\title{
Development of a New Fluorescent Reagent and Its Applica- tion to the Automated Assay of Amino Acids and Peptides at the Picomole Level*
}

\author{
Sidney Udenfriend \\ Roche Institute of Molecular Biology, Nutley, New Jersey 07110
}

(June 15, 1972)

\begin{abstract}
Methods for the assay of amino acids and peptides are most important in elucidating the structure of proteins and peptides. In many important areas of research such as in endocrinology, neurobiology, and genetics, methods are needed with sensitivity higher than is available with the widely used colorimetric ninhydrin procedure. A short while ago, we noted that all primary amines react with ninhydrin and phenylacetaldehyde to give a ternary product which is highly fluorescent. The chemistry of that reaction has now been elucidated and the conditions have been modified and improved so that essentially quantitative yields of fluorescent products are formed with all primary amines. The reaction has been automated and is being used as the detecting system for chromatography of amino acids, peptides and amines in the 10 to 100 picomole range. Problems concerning the fluorescence instrumentation and the isolation and chromatography of these compounds in the picomole range will be discussed.
\end{abstract}

Key words: Fluorometry, amino acids; fluorometry, peptides; picomole fluorometry.

Collaborative studies by biologists and chemists at Nutley have led to the development of a new fluorometric reagent for amino acids and peptides and its application to their automated assay at the picomole level. The origin of these studies, as well as the chemistry and the analytical applications, are presented in this report.

A number of years ago McCaman and Robins $[1]^{1}$ reported a highly specific and sensitive fluorometric method for blood phenylalanine assay. The procedure is now generally used for the diagnosis of phenylketonuria. In this method, fluorescence develops when phenylalanine is treated with ninhydrin and a peptide. The other amino acids which are normally found in plasma and protein hydrolyzates give no fluorescence. On the other hand, almost any peptide can be used in the phenylalanine assay.

These very unusual requirements led us to investigate the reaction with the possibility of reversing the conditions to assay peptides fluorometrically by adding ninhydrin plus phenylalanine. When this was tried it worked. Other than phenylalanine, the only $\alpha$-amino acid which yielded fluorescence (although of far less intensity) was $\alpha$-aminobutyric acid. This amino acid is not normally present in tissues.

On further investigation, it was found that phenylalanine itself was not the active agent, but was first

\footnotetext{
* This article is based on studies carried out in collaboration with Wallace Dairman, Keijiro Samejima, Manfred Weigele, Willy Leimgruber, Stanley Stein, and Peter Böhlen.

${ }^{1}$ Figures in brackets indicate the literature references at the end of this paper.
}

oxidized and decarboxylated by ninhydrin to phenylacetaldehyde. The latter, in the presence of additional ninhydrin, combined with primary aliphatic amines to yield highly fluorescent derivatives. It was found that several other aldehydes yielded fluorophors with ninhydrin and amines but that the most intense fluorescense by far was obtained with phenylacetaldehyde.

The products obtained on reacting amines, amino acids and peptides with ninhydrin and phenylacetaldehyde all exhibit the same excitation and emission spectra (fig. 1). Excitation maxima are at $275 \mathrm{~nm}$ and $390 \mathrm{~nm}$ with emission at $480 \mathrm{~nm}$.

To investigate the mechanism of the reaction further, the fluorophor formed on reacting phenylacetaldehyde and ninhydrin with a simple amine, such as ethylamine, was prepared. The purifled product could not be obtained in crystalline form, but was shown to be in equilibrium with a nonfluorescent congener which was apparently a lactone. The latter was.crystallized and yielded a molecular ion with the highest mass at 305 . This and other findings suggested that the product was the result of a ternary reaction involving one molecule each of amine, phenylacetaldehyde and ninhydrin.

The purified fluorophor obtained with ethylamine was used as a standard to show that under the conditions of the reaction the yield of fluorescent product was about 50 percent. There was considerable variation in the fluorescence obtained with various other amino acids. Much of this was due to differences in quantum yield as well as to completeness of reaction. 


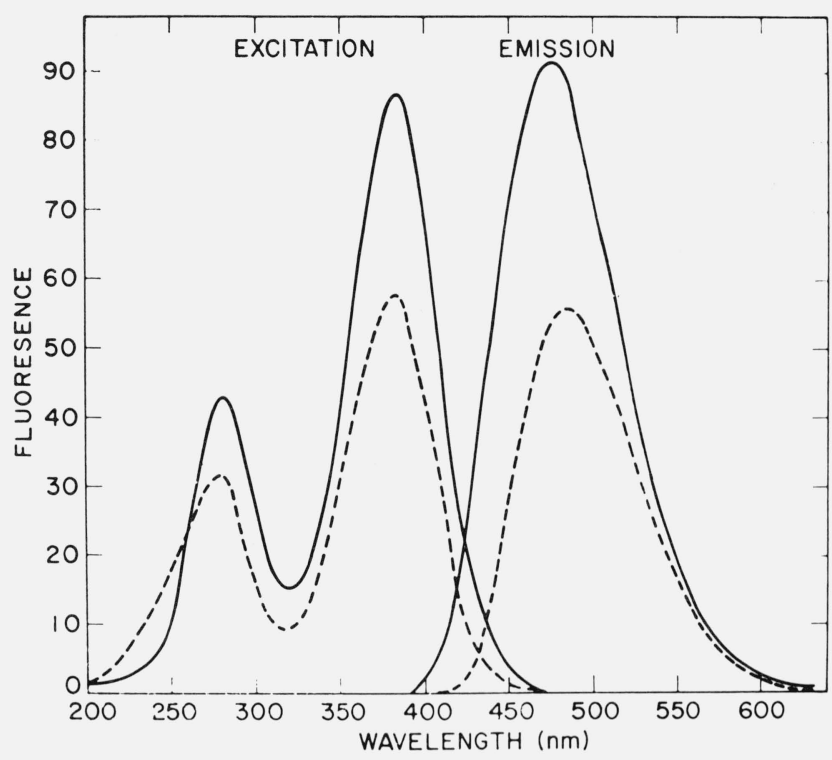

FIGURE 1. Spectral properties of fluorophor obtained on reacting an amino acid with ninhydrin and phenylacetaldehyde.

The procedure was automated with the use of appropriate pumps and a flow cell cuvette in an Aminco Microfluorometer. ${ }^{2}$ Application of this automated procedure was made for amino acid analysis. The sensitivity at this stage with most amino acids was found to be below the nanomole level. This compares to a limit of 10 nanomoles for most commercial amino acid analyzers which utilize the colorimetric ninhydrin procedure.

Details of the procedure utilizing ninhydrin and phenylacetaldehyde have been published $[2,3]$. It possesses the sensitivity and many other qualities which could have made it a useful analytical procedure. However, elucidation of the mechanism of the reaction [4] led to the development of a new reagent and made it obsolete.

Weigele and Leimgruber and their colleagues at Hoffmann-La Roche in Nutley had been collaborating with us in our studies. They concentrated on the mechanism of the ninhydrin phenylacetaldehyde reaction utilizing mass spectrometry, $x$-ray crystallography and other procedures on a number of crystalline derivatives. The structure of the fluorophor obtained with ethylamine was proven to be as shown in figure 2 . The condensation with ninhydrin and phenylacetaldehyde also yielded two nonfluorescent products which could be converted to the fluorophor by treatment with base.

Brilliant deduction led Weigele and Leimgruber ${ }^{3}$ to synthesize the compound, 4-phenylspiro [furan-2 $(3 \mathrm{H})$, $1^{1}$-phthalan]-3,31-dione, RO 20-7234. This compound was found to react with amines to form the identical

\footnotetext{
${ }^{2}$ In order to adequately describe materials and experimental procedures, it was occasionally necessary to identify commercial products by manufacturer's name or label. In no instances does such identification imply endorsement by the National Bureau of Stand ards, nor does it imply that the particular product or equipment is necessarily the best available for that purpose.

3 Weigle, M., and Leimgruber, W., to be published.
}

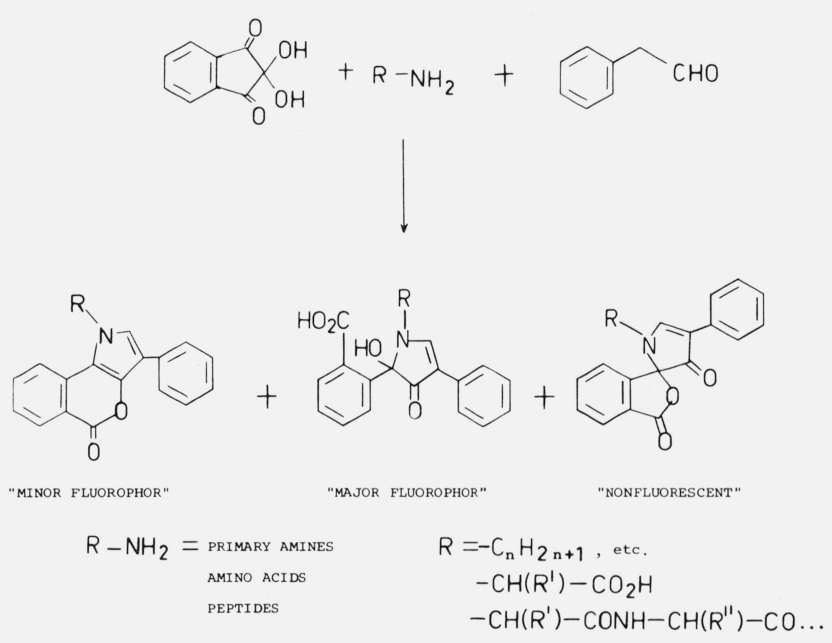

FIGURE 2. Formation of fluorophor from ninhydrin, phenylacetaldehyde and ethylamine.

fluorophor obtained with ninhydrin and phenylacetaldehyde (fig. 3). With this new reagent the reaction is a<smiles>O=C1OC2(OC=C(c3ccccc3)C2=O)c2ccccc21</smiles>

RO $20-7234$
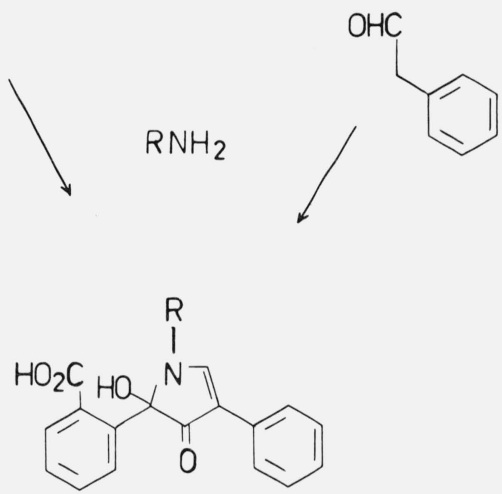

"MAJOR FLUOROPHOR"

FIGURE 3. Comparison of reactions of amines with RO 20-7234 and with ninhydrin and phenylacetaldehyde.

binary one rather than a ternary one. The resulting advantages are many-fold. The ternary reaction with ninhydrin does not go to completion; about 45 percent with ethylamine and much less for aspartic acid. Yields with the new reagent are almost quantitative with all amines, amino acids and peptides. The ternary reaction is run at $65^{\circ}$ for $25 \mathrm{~min}$. The binary reaction is instantaneous at room temperature. In both cases the reagents are nonfluorescent. In the ternary reaction, ammonia does not react at all. With the new reagent, ammonia reacts to a slight extent (one thousandth as 
much as a typical amino acid). The conditions of the binary reaction lead to several times more intense fluorescence for the same amount of fluorophor.

The fluorophor obtained with peptides (fig. 4) are
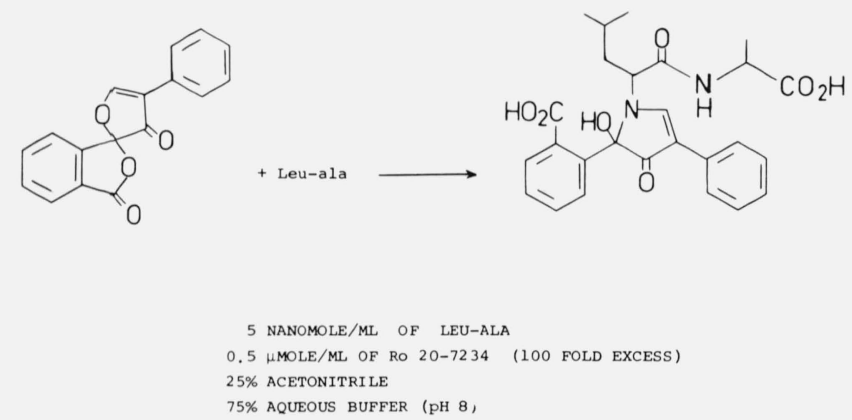

Figure 4. Formation of fluorophor with a peptide and the reagent RO 20-7234.

more intensely fluorescent than the fluorophors formed from the corresponding amino acids. This may be due to a greater quantum yield. The free carboxyl group close to the fluorophoric group may quench the fluorescence. Whatever the reason, this is a decided advantage for peptide assay.

The new reagent is ideally suited to detect amino acids and peptides in the automated assay of column effluents. Such assays have now been set up and even with relatively unsophisticated pumping systems and fluorescence instrumentation, it has been possible to carry out analyses in the picomole range. Figure 5 shows the chromatographic separation of an amino acid mixture (neutral and acidic), 250 picomoles of each. Figure 6 shows the chromatographic separation of 50 picomoles each of the basic amino acids. ${ }^{4}$

A number of applications have already been made which make use of this great sensitivity. It has been possible to measure the peptides oxytocin and vasopressin in an aliquot of an extract of a single rat pituitary (fig. 7). Thus, the method will be of great
SEPARATION OF AMINO ACIDS

ON A BASICS COLUMN

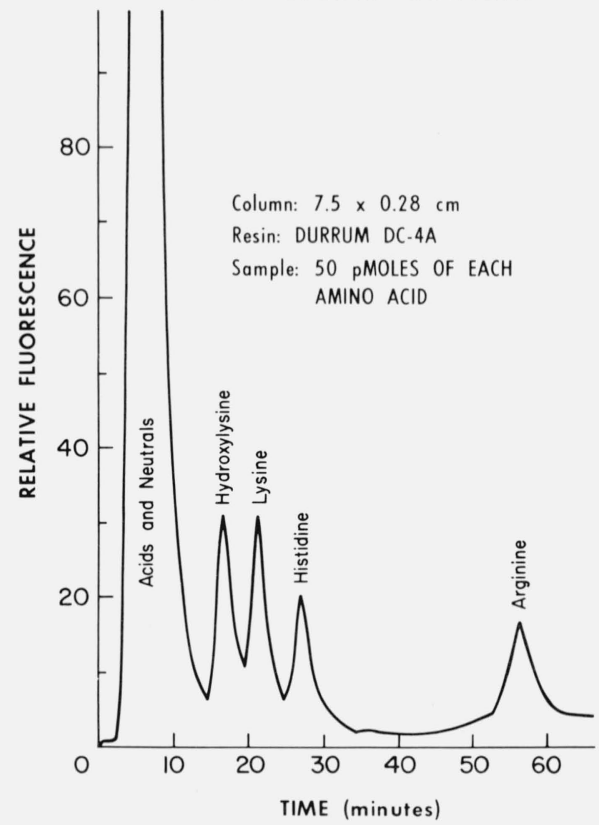

Figure 6. Chromatography of basic amino acids in the picomole range.

importance in the isolation and characterization of physiologically active peptides and their metabolites. Another important application has been to the assay of spermine and spermidine. The sensitivity in this case is so great and the tissue levels so high that microgram quantities of brain tissue are sufficient for assay. Most important, the simplicity of the apparatus required to operate in the picomole region using fluorescence should make it possible to devise relatively inexpensive commercial equipment. It is hoped that commercial instruments, which make use of this reagent, will soon be available.

\section{CHROMATOGRAPHY OF ACIDIC AND NEUTRAL AMINO ACIDS}

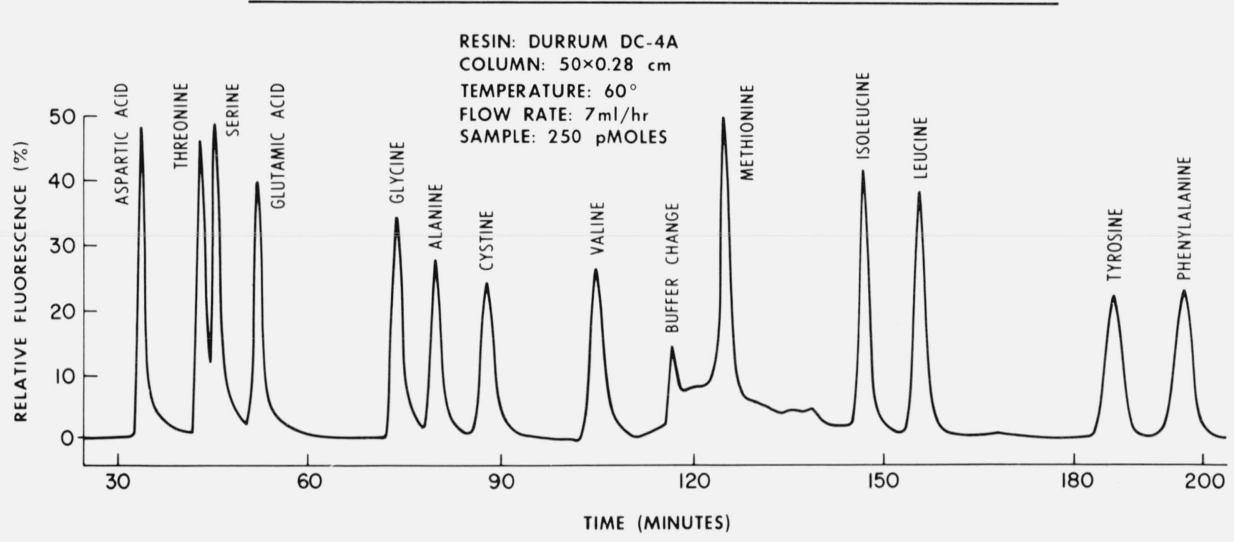

Figure 5. Chromatography of neutral and acidic amino acids in the picomole range.

\footnotetext{
${ }^{4}$ Details of these procedures will be published in a subsequent paper.
} 
PROFILE OF $500 \sim 10,000$ M.W.

ON CM CELLULOSE 2-17-71

STD: I/3 OF COLUMN ALIQUOT

त COLUMN ALIQUOT: EQUIVALENT

TO I/2 OF I RAT PITUITARY

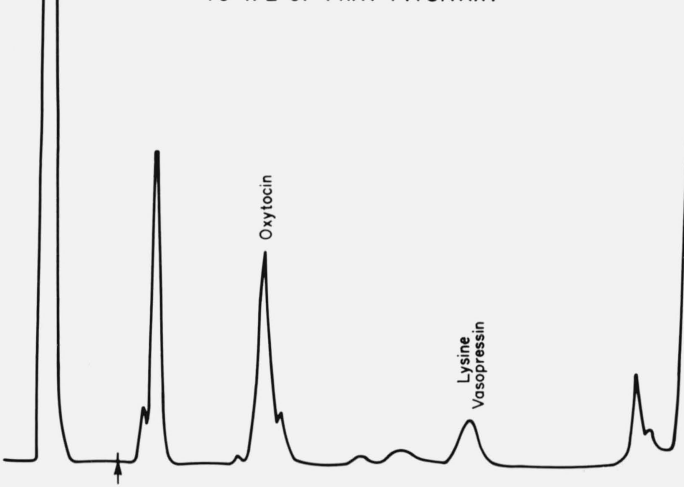

STD START

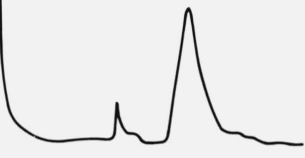

FIGURE 7. Chromatographic separation of the oxytocin and vasopressin in an extract of one rat pituitary.

\section{References}

[1] McCaman, M. W., and Robins, E., J. Lab. Clin. Med. 59, 885 (1962).

[2] Samejima, K., Dairman, W., and Udenfriend, S., Anal. Biochem. 42, 222 (1971).
[3] Samejima, K., Dairman, W., Stone, J., and Udenfriend, S., Anal. Biochem. 42, 237 (1971).

[4] Weigele, M., Blount, J. F., Tengi, J. P. Czajkowski, R. C., and Leimgruber, W., J. Am. Chem. Soc. 94, 4052 (1972).

(Paper 76A6-747) 